Nota de opinión

\title{
Diferencias conceptuales entre modelación de nichos y modelación de áreas de distribución
}

\author{
Conceptual differences between ecological niche modeling and species distribution modeling \\ Jorge Soberón $^{\mathrm{a}, *}$, Luis Osorio-Olvera ${ }^{\mathrm{b}}$ y Townsend Peterson ${ }^{\mathrm{a}}$ \\ ${ }^{a}$ Departamento de Ecología y Biología Evolutiva e Instituto de Biodiversidad, Universidad de Kansas, 1345 Jayhawk Blvd., Lawrence, KS 66045, Estados Unidos \\ ${ }^{\mathrm{b}}$ Facultad de Ciencias, Universidad Nacional Autónoma de México, Circuito Exterior s/n, Cd. Universitaria, 04510 Ciudad de México, México
}

\section{Introducción}

La disciplina, que puede considerarse parte de la macroecología o la biogeografía, de modelar las áreas de distribución de las especies utilizando los así llamados «modelos de nicho», ha crecido explosivamente en los últimos 20 años (Guisan et al., 2013). Una característica curiosa de esta nueva disciplina, nueva relativamente, ya que hay antecedentes desde la década de 1970 (Soto, Giddings y Gómez, 1996), es el predominio de resultados prácticos sobre la claridad conceptual (McInerny y Etienne, 2012). La abundancia de datos de presencias (Gaiji et al., 2013), la disponibilidad de datos de coberturas climáticas mundiales (e.g., Hijmans, Cameron, Parra, Jones y Jarvis, 2005) o nacionales (e.g., Cuervo-Robayo et al., 2014), y la variedad de programas de cómputo que correlacionan presencias con coberturas ambientales para estimar mapas (Franklin, 2009) han permitido una explosión en el número de aplicaciones, algunas de las cuales pueden adolecer de problemas conceptuales.

Un ejemplo de lo anterior es la extendida costumbre de considerar «modelación de nichos (ENM)» y «modelación de áreas de distribución (SDM)» como sinónimos (Elith y Graham, 2009; Elith y Leathwick, 2009). Estos 2 términos no son equivalentes y percibirlos como tales lleva a confusión y problemas de interpretación de resultados. La aplicación de métodos correlativos a puntos de presencias de una especie para identificar variables ambientales asociadas permite sin duda crear mapas que se pueden interpretar de diversas maneras, incluyendo distribuciones, potenciales o actuales, de una especie. Pero independientemente del aspecto estrictamente semántico, existe una clara diferencia entre modelar nichos o modelar distribuciones (Peterson y

\footnotetext{
* Autor para correspondencia.

Correo electrónico: jsoberon@ ku.edu (J. Soberón).

La revisión por pares es responsabilidad de la Universidad Nacional Autónoma de México.
}

Soberón, 2012; Warren, 2012), y el propósito de esta opinión es discutir y ejemplificar esta diferencia. Insistimos en que el punto no es meramente semántico, sino conceptual y está en la raíz de las disciplinas de ENM y SDM.

\section{La «dualidad de Hutchinson»}

Una forma clara y gráfica de discutir la diferencia entre ENM y SDM es utilizar la llamada «dualidad de Hutchinson» (Colwell y Rangel, 2009), que es una hipótesis propuesta por Hutchinson (1957) sobre la correspondencia entre el espacio geográfico y el espacio de nicho, abstracto y multidimensional. En la figura 1 se ilustra esta dualidad.

Esta correspondencia se hace evidente y se puede operacionalizar (Hijmans, Phillips, Leathwick, Elith y Hijmans, 2015) cuando se impone una retícula de resolución dada, con una proyección específica, sobre la región de interés y se procede a obtener los valores de las variables usadas para el espacio de nicho mediante coberturas ambientales en formato «raster» (Hijmans et al., 2015; Peterson et al., 2011). Si bien a cada punto de la retícula geográfica (que llamaremos G) corresponde un único punto del espacio ambiental (que llamaremos E), lo contrario no es verdad necesariamente porque puede haber 2 regiones del planeta con valores de las variables ambientales iguales o similares. Esta posibilidad en la práctica desaparece cuando se usan suficientes variables, con la precisión adecuada (AielloLammens, Boria, Radosavljevic, Vilela y Anderson, 2015). En este caso se establece una relación 1:1 entre los puntos del espacio $\mathrm{G}$ y los del espacio $\mathrm{E}$. La modelación correlativa de nichos permite entonces crear un objeto en $\mathrm{E}$ que es posteriormente «proyectado» a G, como se describe abajo. Es importante reconocer, no obstante, que estos espacios tienen topologías muy diferentes, que puntos muy lejanos en el espacio geográfico pueden ser muy similares en el espacio ambiental y que puntos muy 
Espacio ambiental (E)

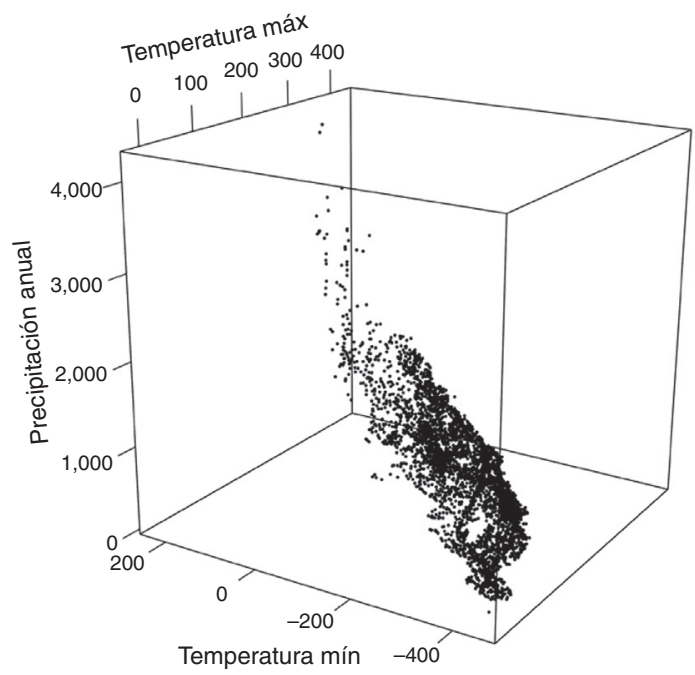

Espacio geográfico (G)

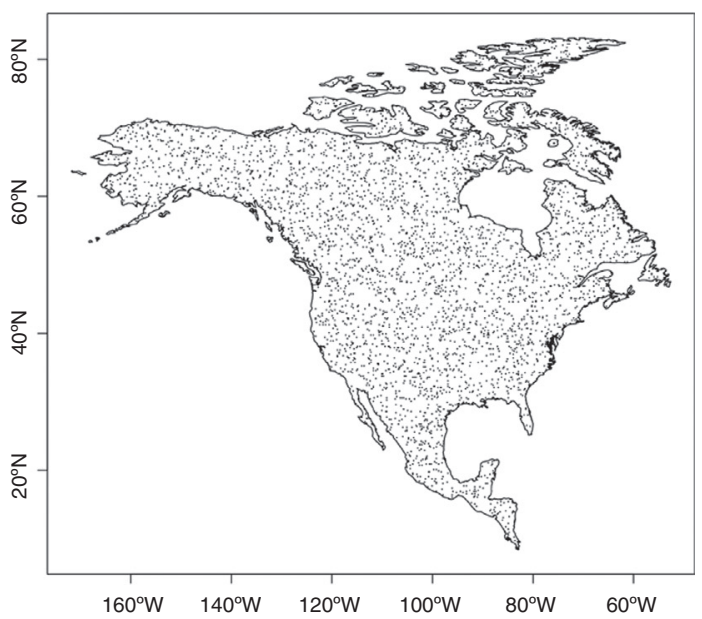

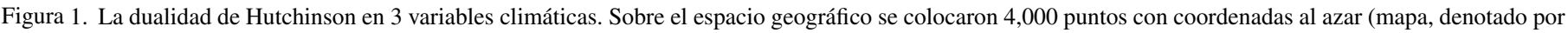

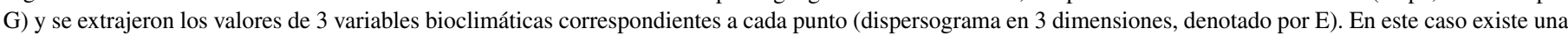
correspondencia 1 a 1 entre puntos de $\mathrm{E}$ y de G.

cercanos en el espacio geográfico pueden ser muy diferentes en el ambiental.

La primera y obvia razón, entonces, por la cual SDM y ENM son diferentes, es que las áreas de distribución son subconjuntos del espacio G, mientras que los nichos son objetos en $\mathrm{E}$. A despecho de que exista una relación 1:1 entre $\mathrm{G}$ y E, se trata de objetos esencialmente diferentes, que «habitan» espacios muy distintos, con propiedades topológicas diferentes. Sin embargo, existe una razón aún más fundamental para establecer la diferencia entre ENM y SDM.

\section{EI diagrama BAM}

Entre los factores que determinan causalmente el área de distribución de una especie, se encuentran sus tolerancias ambientales (su nicho fundamental), la presencia o ausencia de otras especies (polinizadoras, dispersoras, competidoras, depredadoras, etc.) y las posibilidades de dispersión en períodos de tiempo y desde áreas originales relevantes (Cain, 1944; Gaston, 2003; Good, 1931; Udvardy, 1969). Dicho en otras palabras, un área de distribución, en general, depende de muchos factores, más que el simple nicho fundamental de la especie. Una manera muy simplificada de expresar lo anterior es el llamado «diagrama BAM» (Soberón y Peterson, 2005), ilustrado en la figura 2.

El diagrama BAM muestra que una especie tiene altas probabilidades de ser observada en aquellas regiones del planeta donde: i) existan las condiciones abióticas necesarias, simbolizadas por «A»; ii) existan las condiciones bióticas favorables $(« \mathrm{~B} »)$, en otras palabras, el medio biótico de la especie (McGill, Enquist, Weiher y Westoby, 2006) y iii) hayan estado accesibles a la dispersión de la especie desde la región de su origen histórico, o bien desde alguna región definida en un momento temporal relevante $(« \mathrm{M} »)$, esto es, la región del planeta accesible a la dispersión (Svenning y Skov, 2004), lo cual es un asunto esencialmente histórico (Hortal, Lobo y Jiménez-Valverde, 2012). Por ejemplo, qué partes de Norteamérica han estado accesibles desde el último máximo glacial, a partir del Eje Neovolcánico. La zona denotada por $\mathrm{G}_{O}$, o área ocupada es la intersección de favorabilidad biótica y abiótica, y de accesibilidad histórica. La especie puede ser observada/recolectada en esa región.

Por definición, las variables ambientales en $\mathrm{G}_{O}$ constituyen el nicho realizado $\left(\mathrm{N}_{R}\right)$. La zona $\mathrm{G}_{I}$ es una región con condiciones favorables pero que se ha mantenido fuera de las capacidades de dispersión de la especie. La región A es favorable a la especie si se hace abstracción de las interacciones y por lo tanto su

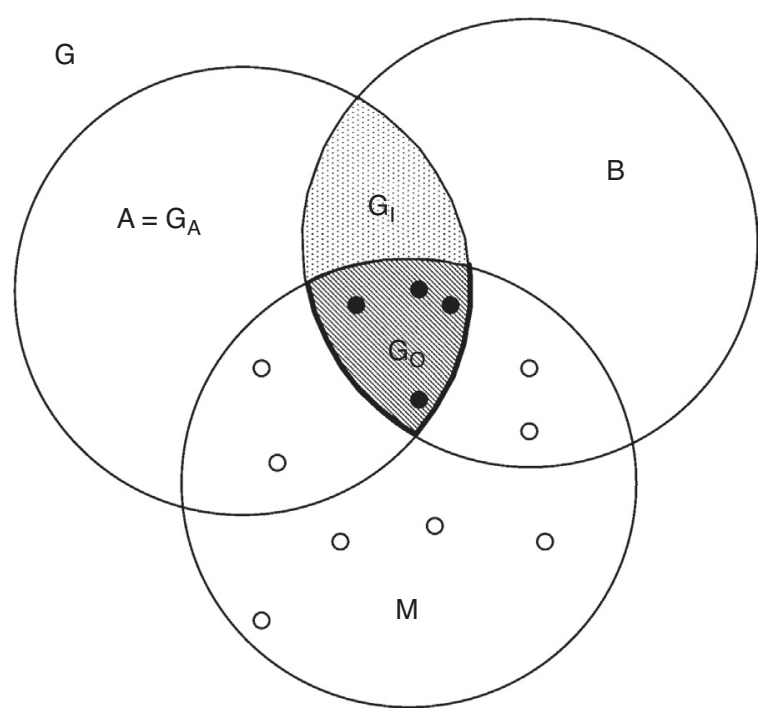

Figura 2. El diagrama BAM es una representación idealizada del espacio geográfico $\mathrm{G}$, donde se ilustran diferentes regiones. Los puntos rellenos representan observaciones de poblaciones «fuente», denotadas por $\mathrm{G}_{+}$y que por ser observaciones dependen de la calidad del muestreo. Los círculos abiertos son poblaciones «sumidero». Cualquier algoritmo correlativo trabaja sobre estos puntos, abiertos y llenos. 
a

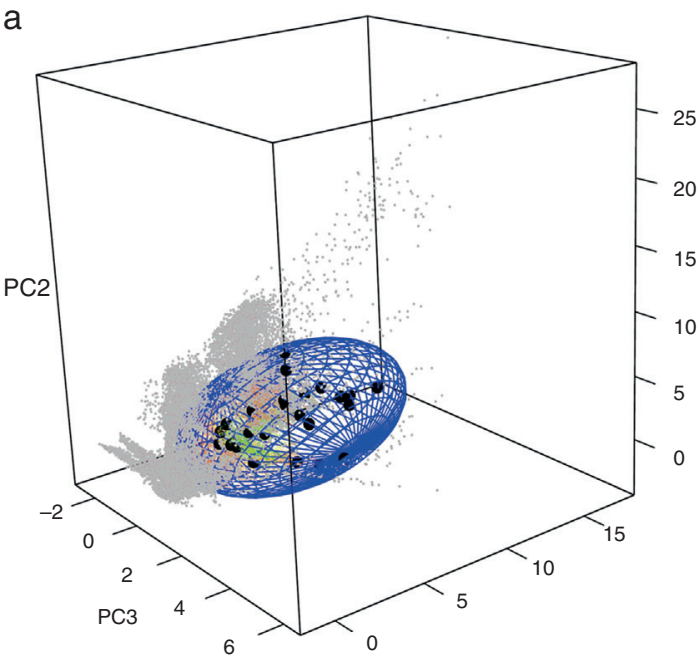

b

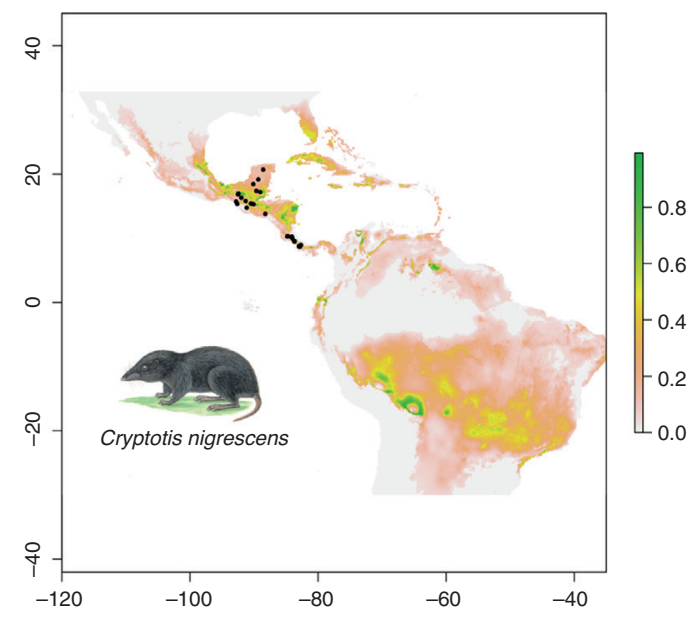

Figura 3. Modelo de nicho (elipsoide, fig. 3a) del complejo C. nigrescens y su proyección en la geografía, o modelo de distribución (mapa, fig. 3b). Los puntos grises corresponden a los sitios con ambientes fuera del nicho de la especie. Los círculos en negro, tanto en el espacio de nicho como en el mapa, son las observaciones procedentes de GBIF. Los puntos en el espacio de nicho son más idóneos mientras más cerca estén del centroide de la elipse. En la versión a color de la figura, un color verde indica cercanía al centroide (alta idoneidad ambiental) y un color rojo, lejanía del centroide (baja idoneidad ambiental).

medio ambiente corresponde a la idea de «nicho fundamental» de Hutchinson (1957), aunque más específicamente, a la parte del nicho fundamental que existe en una región dada, en un período dado (Jackson y Overpeck, 2000) y que simbolizamos con $\mathrm{N}_{F}^{*}$, o el nicho existente. Las relaciones entre nichos y áreas se describen en la tabla 1 y se detallan en Peterson et al. (2011).

Resulta entonces que cuando se utilizan algoritmos correlativos para modelar distribuciones de especies, el algoritmo combina las presencias y las capas ambientales para generar un objeto (tabla, fórmula, conjunto) en el espacio E, que es posteriormente proyectado a $\mathrm{G}$ como consecuencia de la dualidad de Hutchinson (Guisan y Zimmermann, 2000; Peterson et al., 2011). Sin embargo, en los modelos correlativos el investigador muy raramente incluye explícitamente las capas B y M; algunas excepciones son Araújo y Luoto (2007) y Heikkinen, Luoto, Virkkala, Pearson y Korber (2007). Así, en una modelación correlativa, ni las interacciones ni los factores históricos y de dispersión son tomados en cuenta explícita y directamente (Barve et al., 2011). En este caso se modelan los ambientes A, y la proyección a $\mathrm{G}$ es una distribución potencial (Peterson, Papeş y Soberón, 2015). Vemos entonces que SDM se refiere a modelar objetos en G. Estos pueden ser considerados áreas potenciales $\left(\mathrm{A}\right.$ o $\mathrm{G}_{I}$ ), si se ignoran los efectos de $\mathrm{B}$ y $\mathrm{M}$, o áreas ocupadas $\left(\mathrm{G}_{O}\right)$, si se cuenta con información sobre B y M. A su vez, ENM se refiere a modelar condiciones ambientales, que se aproximan más o menos a $\mathrm{N}_{F}^{*}$ o a $\mathrm{N}_{R}$ dependiendo del algoritmo, de la

Tabla 1

Relaciones entre nichos y áreas.

\begin{tabular}{ll}
\hline Espacio G & Espacio E \\
\hline A (área potencial) & $\mathrm{N}_{F}$ (nicho existente) \\
$\mathrm{G}_{o}$ (área ocupada) & $\mathrm{N}_{R}$ (nicho realizado) \\
$\mathrm{G}_{i}$ (área invadible) & Nicho realizado acotado por movimientos \\
Observaciones & Modelo de nicho. Probablemente intermedio \\
& entre $\mathrm{N}_{F} \mathrm{y} \mathrm{N}_{R}$ \\
\hline
\end{tabular}

configuración del BAM y de la calidad de las observaciones (Qiao, Soberón y Peterson, 2015; Saupe et al., 2012). Obtener aproximaciones al $\mathrm{N}_{F}$ es otro problema y no lo discutiremos en esta breve nota.

Para ilustrar lo anterior, usamos como ejemplo un complejo de 3 especies de musaraña, Cryptotis nigrescens sensu lato (el complejo incluye $C$. mayensis, $C$. merriami y $C$. nigrescens; fig. 3), con 31 observaciones obtenidas del Global Biodiversity Information Facility. Usando un algoritmo de elipsoides de volumen mínimo (Osorio-Olvera, Barve, Barve y Soberón, 2016) estimamos un ENM para $C$. nigrescens. Este ENM está en el
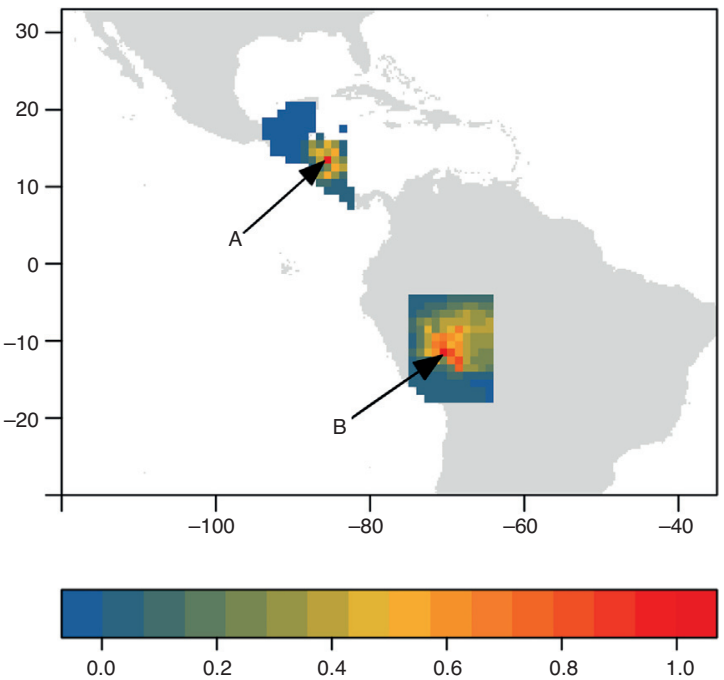

Figura 4. Dos predicciones del área de distribución a partir de 2 puntos de inicio diferentes. A, es un inicio cercano al área ocupada de la especie (puntos negros en la figura 3b); B, es un inicio muy lejano al área original, simulando la introducción de la especie a Brasil. La escala de colores representa la densidad relativa (respecto al máximo, en rojo) de individuos. En la versión en blanco y negro, las densidades máximas se encuentran hacia el centro de las 2 regiones del mapa. 
espacio E, representado por el elipsoide azul de la figura 3a. La proyección del modelo en el espacio geográfico (fig. 3b) muestra 2 áreas favorables a la especie, una en Centroamérica, cercana a los puntos de observaciones, y otra en Sudamérica, donde la especie no existe. Las hipótesis sobre $\mathrm{M}$ se pueden explorar usando modelos basados en procesos (Barve et al., 2011; Osorio-Olvera, Falconi y Soberón, 2016; Schurr et al., 2012). Una hipótesis que restringiera la $\mathrm{M}$ a la región donde el complejo de $C$. nigrescens ha existido históricamente produciría un SDM en Centroamérica. Otra hipótesis basada en una hipotética invasión de la especie a Brasil crearía un SDM completamente distinto, en el occidente de Brasil (fig. 4).

\section{Conclusión}

En el trabajo científico es esencial utilizar rigurosamente la terminología. Considerar que los modelos de nicho (ENM) y los modelos de áreas (SDM) son lo mismo, o que la terminología es «neutral» (Elith y Leathwick, 2009), conduce únicamente a dificultar la claridad conceptual y la comunicación entre científicos. Es obvio que existe una íntima e interesante relación entre nichos y áreas de distribución, que es lo que permite modelar las segundas mediante los primeros. Sin embargo, no se gana nada considerando los 2 procesos de modelación como sinónimos. Sugerimos que se evite hacerlo y se usen los términos SDM y ENM según resulten apropiados al objeto de cada estudio.

\section{Agradecimientos}

La inspiración para realizar este trabajo se debe en buena medida a nuestros colegas y estudiantes de los múltiples cursos sobre modelación de nicho en los que hemos participado; en particular, pero sin ser exhaustivos, agradecemos a Enrique Martínez-Meyer, Andrés Lira-Noriega, Octavio Rojas, Fabricio Villalobos, Pilar Rodríguez, Carlos Yáñez-Arenas y Manuel Falconi. Agradecemos el apoyo moral de Blitzi Soberón y el apoyo económico parcial de PAPIIT-UNAM IN112715 (2015) y del Posgrado en Ciencias Biológicas de la UNAM para LOO y el de National Science Foundation 1458640 para JS.

\section{Referencias}

Aiello-Lammens, M. E., Boria, R. A., Radosavljevic, A., Vilela, B. y Anderson, R. P. (2015). spThin: an R package for spatial thinning of species occurrence records for use in ecological niche models. Ecography, 38, 541-545.

Araújo, M. B. y Luoto, M. (2007). The importance of biotic interactions for modelling species distributions under climate change. Global Ecology and Biogeography, 16, 743-753.

Barve, N., Barve, V., Jiménez-Valverde, A., Lira-Noriega, A., Maher, S. P., Peterson, A. T., et al. (2011). The crucial role of the accessible area in ecological niche modeling and species distribution modeling. Ecological Modelling, 222, 1810-1819.

Cain, S. A. (1944). Foundations of plant geography. New York: Harper \& Brothers.

Colwell, R. K. y Rangel, T. F. (2009). Hutchinson's duality: the once and future niche. Proceedings of the National Academy of Sciences USA, 106, 19644-19650.

Cuervo-Robayo, A. P., Téllez-Valdés, O., Gómez-Albores, M. A., VenegasBarrera, C. S., Manjarrez, J. y Martínez-Meyer, E. (2014). An update of high-resolution monthly climate surfaces for Mexico. International Journal of Climatology, 34, 2427-2437.

Elith, J. y Graham, C. (2009). Do they? How do they? Why do they differ? On finding reasons for differing performances of species distribution models. Ecography, 32, 66-77.

Elith, J. y Leathwick, J. (2009). Species distribution models: ecological explanation and prediction across space and time. Annual Review of Ecology, Evolution and Systematics, 40, 677-697.

Franklin, J. (2009). Mapping species distributions: spatial inference and prediction. Cambridge: Cambridge University Press.

Gaiji, S., Chavan, V., Ariño, A. H., Otegui, J., Hobern, D., Sood, R., et al. (2013). Content assessment of the primary biodiversity data published through GBIF network: status, challenges and potentials. Biodiversity Informatics, 8, 94-172.

Gaston, K. (2003). The structure and dynamics of geographic ranges. Oxford: Oxford University Press.

Good, R. D. (1931). A theory of plant geography. The New Phytologist, 30, 149-171.

Guisan, A., Tingley, R., Baumgartner, J. B., Naujokaitis-Lewis, I., Sutcliffe, P. R., Tulloch, A. I. T., et al. (2013). Predicting species distributions for conservation decisions. Ecology Letters, 16, 1424-1435.

Guisan, A. y Zimmermann, N. (2000). Predictive habitat distribution models in ecology. Ecological Modelling, 135, 147-186.

Heikkinen, R. K., Luoto, M., Virkkala, R., Pearson, R. G. y Korber, J. H. (2007). Biotic interactions improve prediction of boreal bird distributions at macroscales. Global Ecology and Biogeography, 16, 754-763.

Hijmans, R. J., Cameron, S., Parra, J., Jones, P. G. y Jarvis, A. (2005). Very high resolution interpolated climate surfaces for global land areas. International Journal of Climatology, 25, 1965-1978.

Hijmans, R. J., Phillips, S., Leathwick, J., Elith, J. y Hijmans, M. R. J. (2015). Package 'dismo'. Circles, 9, 1.

Hortal, J., Lobo, J. M. y Jiménez-Valverde, A. (2012). Basic questions in biogeography and the (lack of) simplicity of species distributions: putting species distribution models in the right place. Natureza \& Conservacao, 10, 108-118.

Hutchinson, G. E. (1957). Concluding remarks. Cold Spring Harbor Symposia on Quantitative Biology, 22, 415-427.

Jackson, S. T. y Overpeck, J. T. (2000). Responses of plant populations and communities to environmental changes of the late Quaternary. Paleobiology, 26(Supl.), 194-220.

McGill, B. J., Enquist, B. J., Weiher, E. y Westoby, M. (2006). Rebuilding community ecology from functional traits. Trends in Ecology and Evolution, 21, 179-185.

McInerny, G. J. y Etienne, R. S. (2012). Ditch the niche-is the niche a useful concept in ecology or species distribution modelling? Journal of Biogeography, 39, 2096-2102.

Osorio-Olvera, L., Barve, V., Barve, N. y Soberón, J. (2016). Nichetoolbox: from getting biodiversity data to evaluating species distribution models in a friendly GUI environment, R package version 0.2.0.0. Github.

Osorio-Olvera, L., Falconi, M. y Soberón, J. (2016). Sobre la relación entre idoneidad del hábitat y la abundancia poblacional bajo diferentes escenarios de dispersión. Revista Mexicana de Biodiversidad, 87, 1080-1088.

Peterson, A. T., Papeş, M. y Soberón, J. (2015). Mechanistic and correlative models of ecological niches. European Journal of Ecology, 1, 28-38.

Peterson, A. T. y Soberón, J. (2012). Species distribution modeling and ecological niche modeling: getting the concepts right. Natureza \& Conservacao, 10, 1-6.

Peterson, A. T., Soberón, J., Pearson, R. G., Anderson, R., Martínez-Meyer, E., Nakamura, M., et al. (2011). Ecological niches and geographic distributions: monographs in population biology. Princeton: Princeton University Press.

Qiao, H., Soberón, J. y Peterson, A. T. (2015). No silver bullets in correlative ecological niche modelling: insights from testing among many potential algorithms for niche estimation. Methods in Ecology and Evolution, 6, 1126-1136.

Saupe, E. E., Barve, V., Myers, C. E., Soberón, J., Barve, N., Hensz, C. M., et al. (2012). Variation in niche and distribution model performance: the need for 
a priori assessment of key causal factors. Ecological Modelling, 237-238, $11-22$.

Schurr, F. M., Pagel, J., Cabral, J. S., Groeneveld, J., Bykova, O., O’Hara, R. B., et al. (2012). How to understand species' niches and range dynamics: a demographic research agenda for biogeography. Journal of Biogeography, 39, 2146-2162.

Soberón, J. y Peterson, A. T. (2005). Interpretation of models of fundamental ecological niches and species' distributional areas. Biodiversity Informatics, $2,1-10$.
Soto, M., Giddings, L. y Gómez, M. (1996). Algunos usos de bioclimas: un sistema especializado de información geográfica. Boletín de Investigaciones Geográficas Núm Especial, 4, 63-83.

Svenning, J. C. y Skov, F. (2004). Limited filling of the potential range in European tree species. Ecology Letters, 7, 565-573.

Udvardy, M. (1969). Dynamic zoogeography, with special reference to land animals. New York: van Nostrand Reinhold.

Warren, D. L. (2012). In defense of 'niche modeling'. Trends in Ecology \& Evolution, 27, 497-500. 